\title{
$\alpha$-Synuclein oligomers distinctively permeabilize complex model membranes
}

\author{
Anja N. D. Stefanovic ${ }^{1}$, Martin T. Stöckl ${ }^{1, *}$, Mireille M. A. E. Claessens ${ }^{1}$ and Vinod Subramaniam ${ }^{1, \dagger}$ \\ 1 Nanobiophysics Group, MIRA Institute for Biomedical Technology and Technical Medicine and MESA+ Institute for Nanotechnology, \\ University of Twente, Enschede, The Netherlands
}

\author{
Keywords \\ cardiolipin; model membranes; oligomers; \\ permeabilization; $\alpha$-synuclein

\section{Correspondence \\ V. Subramaniam, FOM Institute AMOLF, Science Park 104, 1098 XG Amsterdam, \\ The Netherlands \\ Fax: +31 207547290 \\ Tel.: +31207547100 \\ E-mail: subramaniam@amolf.nl \\ Present address: \\ *Bioimaging Center, University of Konstanz, Universitätstrasse 10, 78457, Konstanz, \\ Germany \\ †FOM Institute AMOLF, Science Park 104, Amsterdam, The Netherlands}

(Received 31 January 2014, revised 16 March 2014, accepted 23 April 2014)

doi:10.1111/febs.12824
$\alpha$-Synuclein oligomers are increasingly considered to be responsible for the death of dopaminergic neurons in Parkinson's disease. The toxicity mechanism of $\alpha$-synuclein oligomers likely involves membrane permeabilization. Even though it is well established that $\alpha$-synuclein oligomers bind and permeabilize vesicles composed of negatively-charged lipids, little attention has been given to the interaction of oligomers with bilayers of physiologically relevant lipid compositions. We investigated the interaction of $\alpha$-synuclein with bilayers composed of lipid mixtures that mimic the composition of plasma and mitochondrial membranes. In the present study, we show that monomeric and oligomeric $\alpha$-synuclein bind to these membranes. The resulting membrane leakage differs from that observed for simple artificial model bilayers. Although the addition of oligomers to negatively-charged lipid vesicles displays fast content release in a bulk permeabilization assay, adding oligomers to vesicles with compositions mimicking mitochondrial membranes shows a much slower loss of content. Oligomers are unable to induce leakage in the artificial plasma membranes, even after long-term incubation. CD experiments indicate that binding to lipid bilayers initially induces conformational changes in both oligomeric and monomeric $\alpha$-synuclein, which show little change upon long-term incubation of oligomers with membranes. The results of the present study demonstrate that the mitochondrial model membranes are more vulnerable to permeabilization by oligomers than model plasma membranes reconstituted from brainderived lipids; this preference may imply that increasingly complex membrane components, such as those in the plasma membrane mimic used in the present study, are less vulnerable to damage by oligomers.

\section{Introduction}

Parkinson's disease (PD) is one of the most common human neurodegenerative diseases, involving the progressive loss of dopaminergic neurons in the substantia nigra in the midbrain [1]. The appearance of Lewy bodies is characteristic of the pathology of PD. The main component of these Lewy bodies is the protein $\alpha$-synuclein $(\alpha \mathrm{S})$, which is present as amyloid fibrils. $\alpha \mathrm{S}$ comprises a 140 amino acid intrinsically disordered protein [2-7] of as yet unknown function. In PD, aggregation of $\alpha \mathrm{S}$ causes the protein to lose its putative function and gain toxicity [8-11]. $\alpha \mathrm{S}$ consists of a positively-charged $\mathrm{N}$-terminal region (residues 1-60)

\footnotetext{
Abbreviations

BTLEM, brain total lipid extract membrane; CL, cardiolipin; DOPG, 1,2-dioleoylphosphatidylglycerol; GUV, giant unilamellar vesicle; LUV, large unilamellar vesicle; MRE, mean residue ellipticity; PD, Parkinson's disease; PE, phosphatidylethanolamine; POPC, 1-palmitoyl-2oleoylphosphatidylcholine; POPE, 1-palmitoyl-2-oleoylphosphatidylethanolamine; POPG, 1-palmitoyl-2-oleoyl-sn-glycero-3-phosphoglycerol; PS, phosphatidylserine; SUV, small unilamellar vesicle; $\alpha$ S, $\alpha$-synuclein.
} 
containing imperfect KTKEGV repeats, a hydrophobic NAC region (residues 61-95) and a negativelycharged C-terminal region (residues 96-140). For membrane binding, the $\mathrm{N}$-terminal part of $\alpha \mathrm{S}$ plays a key role. Upon binding to phospholipid membranes, the protein adopts an $\alpha$-helical structure $[12,13]$. Interactions of monomeric $\alpha \mathrm{S}$ with lipid vesicles of different compositions and size have been reported [14-19]. Current consensus suggests that intermediate species in the fibril formation pathway of $\alpha \mathrm{S}$ are the toxic species involved in cell death in PD [10,20-22]. Despite structural differences between $\alpha \mathrm{S}$ oligomers and monomers, they both show high affinity for negatively-charged membranes. We have previously shown that oligomer binding induces leakage of artificial negatively-charged lipid vesicles. Although several studies [23-25] have shown that isolated oligomers can decrease the integrity of simple negatively-charged lipid vesicles, little is known about how these species bind and permeabilize natural membranes. Mitochondrial damage has been observed in PD and mitochondrial membranes are therefore considered to be a likely target for oligomer-induced damage [26-28]. Mitochondrial membranes are enriched in cardiolipin [29], a unique negatively-charged diphosphatidylglycerol lipid. Monomeric $\alpha \mathrm{S}$ shows a high binding affinity to cardiolipin-containing membranes [30,31]. Quantitative correlation between $\alpha \mathrm{S}$ in mitochondria and cytosol confirmed that monomeric $\alpha \mathrm{S}$ interacts with mitochondrial membranes [32]. Moreover, it has been reported that $\alpha \mathrm{S}$ is present on mitochondrial membranes in functional dopaminergic neurons of the substantia nigra $[33,34]$, where, at physiological concentrations, $\alpha \mathrm{S}$ is mainly localized at the mitochondrial inner membrane and only a small fraction is found at the outer membrane [32,35]. However, a contrasting report suggests that $\alpha \mathrm{S}$ is localized in the outer mitochondrial membrane [27]. Mitochondria of dopaminergic neurons in brains of PD patients contain a higher concentration of $\alpha \mathrm{S}$ than normal brains [36]. A recent study has suggested that wild-type $\alpha \mathrm{S}$ is mainly localized in mitochondria-associated endoplasmic reticulum membranes and modulates the morphology of mitochondria [37]. Besides these membranes, the plasma membrane has been indicated as a site of oligomer-induced damage. Upon internalization, $\alpha \mathrm{S}$ colocalizes with the inner leaflet of the plasma membrane $[38,39]$. In a yeast model, it was found that $\alpha \mathrm{S}$ binds to plasma membranes [40]. Finally, a recent report suggests that $\alpha \mathrm{S}$ can interact with lipids in the plasma membrane, increasing the membrane permeability as a potential mechanism of extracellular neurotoxicity [41].
In the present study, we investigate the binding of specific, well-characterized, oligomeric $\alpha S$ species to lipid membranes made of physiologically relevant mitochondrial and plasma membrane lipid mixtures. We show that these oligomers cause slow permeabilization of mitochondrial inner membrane mimics, whereas they bind to (but could not induce leakage in) plasma membrane inner leaflet model systems.

\section{Results}

\section{Binding of $\alpha \mathbf{S}$ monomers to bilayers that mimic the lipid composition of natural membranes}

Electrostatic interactions play a key role in binding of monomers to negatively-charged membranes. Membrane binding of monomers is mainly associated with positive charges on the $\mathrm{N}$-terminal part of the protein [19]. Upon binding to negatively-charged lipid bilayers, monomers adopt an amphipathic $\alpha$-helical structure. Fluorescently labeled monomers colocalize with the control 1,2-dioleoylphosphatidylglycerol (DOPG) vesicles (Fig. 1A) and with giant unilamellar vesicles (GUVs) composed of both the mitochondrial and plasma membrane mimicking lipid mixtures (Fig. 1B,C). Analysis of the Alexa 488-fluorescence intensity at GUV membranes showed that the highest amount of monomers was bound at DOPG membranes (Fig. 1D). Unexpectedly, not all brain phosphatidylethanolamine (PS) : brain phosphatidylethanolamine (PE) : cholesterol vesicles bound monomers, with approximately $50 \%$ of the vesicles not showing any colocalization of labeled monomers and GUVs. It should be noted that only vesicles that bind $\alpha S$ were taken into account in in Fig. 1D.

CD spectroscopy was used to assess whether binding of monomers to vesicles of all lipid compositions resulted in conformational changes in the protein. Monomers in solution showed CD spectra typical for an intrinsically disordered protein with a negative peak at $198 \mathrm{~nm}$. As previously reported, $\alpha \mathrm{S}$ monomers form an $\alpha$-helix upon binding to small unilamellar vesicles (SUVs) $[13,42]$. Upon binding membranes mimicking plasma or mitochondrial lipid compositions, monomers adopt an $\alpha$-helical structure that is characterized by two negative bands at 208 and $222 \mathrm{~nm}$ in the CD spectrum. The $\alpha$-helical content of the protein upon binding was estimated from the mean residue ellipticity (MRE) at $222 \mathrm{~nm}$ as explained in the Materials and methods. Binding of monomers to control DOPG vesicles resulted in an $\alpha$-helical content of $65.1 \pm 1.2 \%$. Monomers binding to cardiolipin (CL) : 1-palmitoyl-2-oleoylphosphatidylethanolamine (POPE) : 1-palmitoyl-2-oleoylphosphatidylcholine (POPC) membranes or membranes 

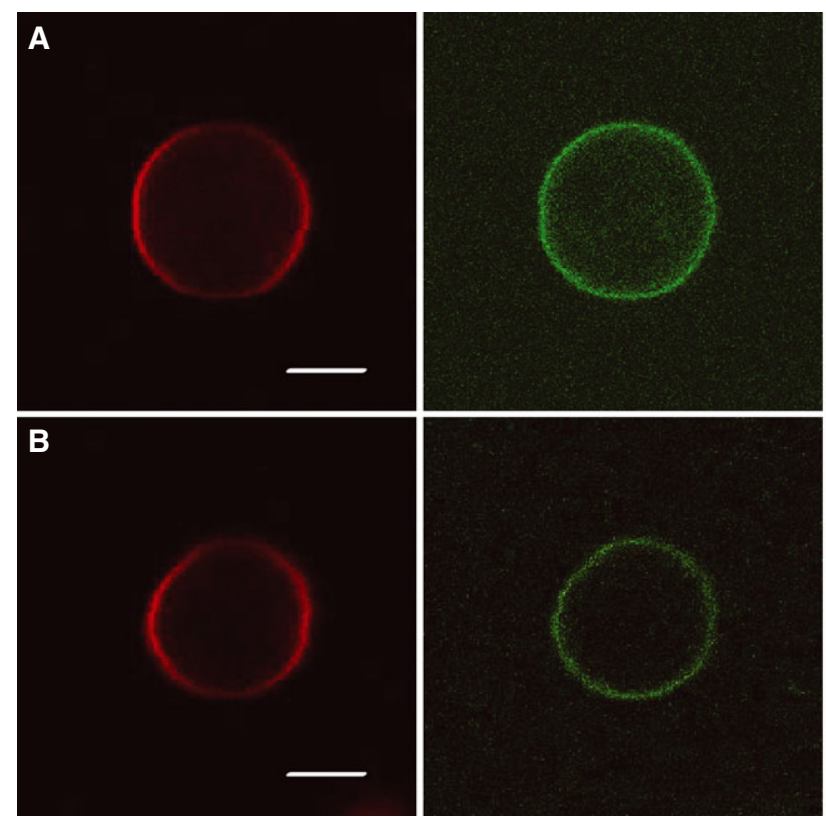
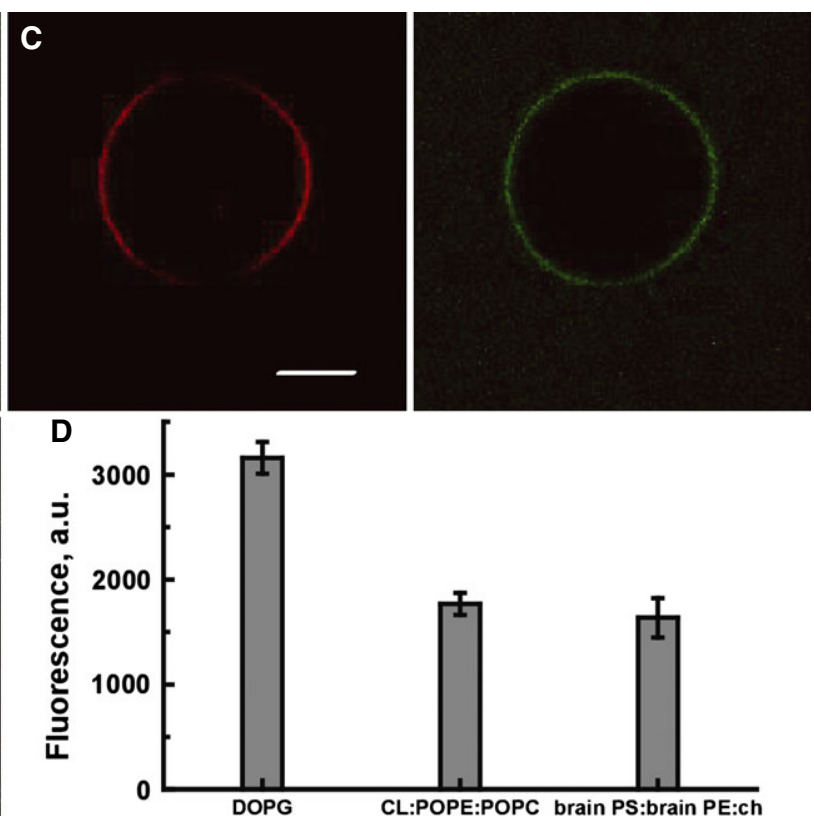

Fig. 1. Representative confocal microscopy images of DOPE-rhodamine labeled GUVs (red channel) and $\alpha \mathrm{S}$ wild-type-140C-A488 monomers (green channel). Binding of $\alpha S$ monomers to (A) DOPG, (B) CL : POPE : POPC $(4: 3: 5)$ and (C) brain PS : brain PE : cholesterol (2: $5: 3$ ) membranes is depicted. (D) Semiquantitative analysis of fluorescently labeled $\alpha \mathrm{S}$ bound to vesicles $(n=15)$. The error bars represent the $\mathrm{SD}$. Scale bars $=5 \mu \mathrm{m}$. The same experimental settings (master gain, digital offset and laser power) were used for all images.

composed of brain PS : brain PE : cholesterol adopted a comparable amount of $\alpha$-helix $(59.0 \pm 0.4 \%$ and $61.1 \pm 0.3 \%$, respectively) (Fig. 2). The similarity of the $\mathrm{CD}$ spectra suggests that $\alpha \mathrm{S}$ monomers were fully bound and in a predominantly $\alpha$-helical conformation at saturating lipid concentrations for all types of natural membranes tested. There were no changes in $\alpha$-helical content of $\alpha \mathrm{S}$ monomers bound to brain PS : brain PE : cholesterol vesicles when comparing CD spectra immediately after binding or after $20 \mathrm{~h}$ of incubation (Fig. 2A). CD data points below $205 \mathrm{~nm}$ were not taken into account because of the high noise, likely as a result of the presence of some other organic compounds in the lipid mixtures of brain lipids.

\section{Do $\alpha \mathrm{S}$ oligomers bind to bilayers mimicking the lipid composition of natural membranes and does this binding result in conformational changes?}

We have previously shown that $\alpha \mathrm{S}$ oligomers can bind to lipid membranes composed of negatively-charged lipids such as DOPG or POPG [43]. The binding of fluorescently labeled oligomers to GUVs with a lipid composition mimicking either negatively-charged, neuronal or mitochondrial membranes was studied using confocal microscopy. We observed that oligomers bind to bilayers mimicking the lipid composition of both the mitochondrial inner membrane and the plasma membrane inner leaflet (Fig. 3B,C). A semi-quantitative comparison of oligomer membrane binding was carried out in accordance with that used for the monomers. Oligomers showed comparable binding to DOPG, CL : POPE : POPC and brain PS : brain PE : cholesterol GUVs (Fig. 3D). Similar to the results observed for monomer binding, we also observed that not all brain PS : brain PE : cholesterol membranes bound oligomers.

We further investigated whether the membrane binding caused conformational changes in the oligomers similar to those observed for monomeric $\alpha \mathrm{S}$ using $\mathrm{CD}$ spectroscopy. In solution, oligomers showed some $\beta$ sheet conformation (Fig. 4). Binding of oligomers to vesicles resulted in a small change in protein conformation for both lipid mixtures. Oligomers showed an increase in $\alpha$-helical content upon the addition of SUVs. The $\alpha$-helical content of the oligomers was comparable for all lipid membranes studied and did not change over time. Oligomers bound to brain PS : brain PE : cholesterol membranes showed similar CD spectra immediately after the addition of protein to membranes and after $20 \mathrm{~h}$ of incubation (Fig. 4).

We further investigated the membrane disrupting properties of oligomers by studying the kinetics of membrane permeabilization using a dye release assay. 

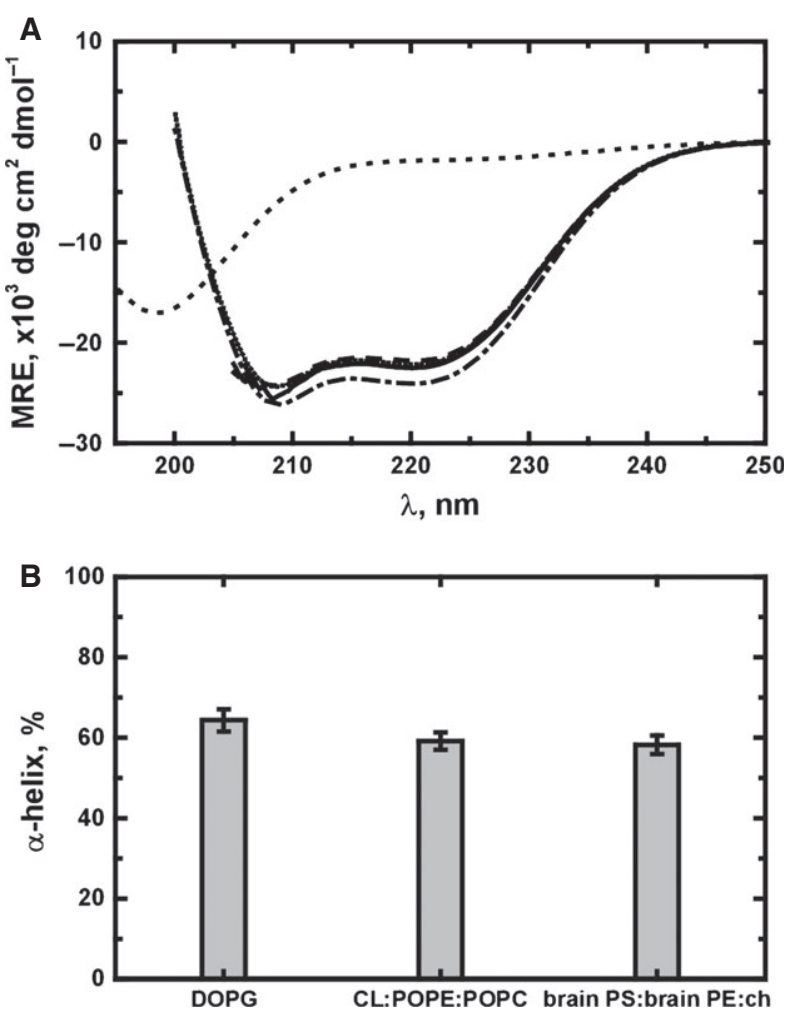

Fig. 2. $C D$ measurements of conformational changes of $\alpha S$ monomers (...) upon binding to membranes. (A) Binding of $\alpha \mathrm{S}$ to DOPG (-.-), CL : POPE : POPC (...) and brain PS : brain $\mathrm{PE}$ : cholesterol (- - ) vesicles showed an increase of $\alpha$-helical content. There is no change in $\alpha$-helical content of $\alpha S$ monomers bound to brain PS : brain PE : cholesterol vesicles over time when comparing $C D$ spectra $10 \mathrm{~min}(--)$ after binding or after $20 \mathrm{~h}$ of incubation (-). (B) Percentage of $\alpha$-helix formed for $\alpha$ S monomers bound to vesicles with the lipid compositions tested above. Data are the mean of three different measurements. Error bars represent the SD.

\section{Kinetics of membrane permeabilization (dye release assay)}

Oligomers have been shown to disrupt membranes composed of negatively-charged lipid vesicles [23]. After the addition of oligomers to a solution of calcein-filled DOPG large unilamellar vesicles (LUVs), a rapid release of encapsulated dye was observed (Fig. 5A). We have previously shown that monomers and fibrils could also cause membrane permeabilization of negatively-charged PG vesicles, although much higher concentrations of the protein are necessary to achieve comparable leakage [23]. A comparison of the oligomer-induced leakage kinetics of the dye from DOPG LUVs with dye encapsulated in vesicles of lipid bilayers that mimic physiologically relevant membrane compositions revealed large differences. Although oligomer binding was observed after 30 min of incubation, very little oligomer-induced vesicle leakage of membranes that mimic natural membranes was observed at these time scales. For LUVs composed of a mixture of CL : POPE : POPC, a slow dye leakage was observed, which was still increasing after $18 \mathrm{~h}$ (Fig. 5B). For these LUVs, the oligomer-induced leakage was concentration-dependent; higher oligomer concentrations showed more leakage. By contrast, even after $18 \mathrm{~h}$ of incubation, oligomer concentrations as high as $4 \mu \mathrm{M}$ did not result in more than $2 \%$ membrane leakage for bilayers composed of brain PS:brain PE:cholesterol (Fig. 5C). To obtain a better understanding of the observed slow release kinetics, we used the diffusion equation assuming steady-state release to fit the data. In the absence of oligomers the change in concentration, $c$, of dye in the vesicles was assumed to follow the expression $\left.c=c(0)\left[1-\mathrm{e}^{-\mathrm{kt}}\right)\right]$ where the rate constant is $k=P_{\mathrm{c}} \times A / V, V$ is the volume of the vesicle, $P_{\mathrm{c}}$ is the membrane permeability for calcein and $A$ is the area over which diffusion takes place. To fit the data in presence of oligomers, we expect the rate constant to change or more exponents to be required to better describe the dye release kinetics.

When oligomers were added, fast leakage was observed for DOPG LUVs. This is not the case for membranes composed of CL : POPE : POPC. Oligomer-induced leakage from these vesicles required a fit with at least three exponentials. One of these exponents is comparable for all oligomer concentrations (the exponent associated with amplitude $C$ in Table 1). This amplitude $(C)$ decreases with increasing oligomer concentration and was attributed to the calcein permeability of the bare bilayer. With increasing oligomer concentration, a decreasing fraction of vesicles is not affected by oligomers and shows leakage comparable to bare membranes. From the slow exponent attributed to the bare membrane, the calcein permeability $P_{\mathrm{c}}$ of the CL : POPE : POPC membrane was calculated to be approximately $10^{-13} \mathrm{~cm} \cdot \mathrm{s}^{-1}$. The amplitudes of the other two exponents increase with increasing oligomer concentration, indicating that these contributions result from the presence of oligomers. Both oligomer-related rate constants were observed to increase with increasing oligomer concentration (Table 1).

\section{Discussion}

It is well established that binding of $\alpha \mathrm{S}$ to lipid bilayers is accompanied by an increase in $\alpha$-helical content [13]. The binding of $\alpha \mathrm{S}$ to lipid bilayers strongly depends on the presence of negatively-charged lipids 

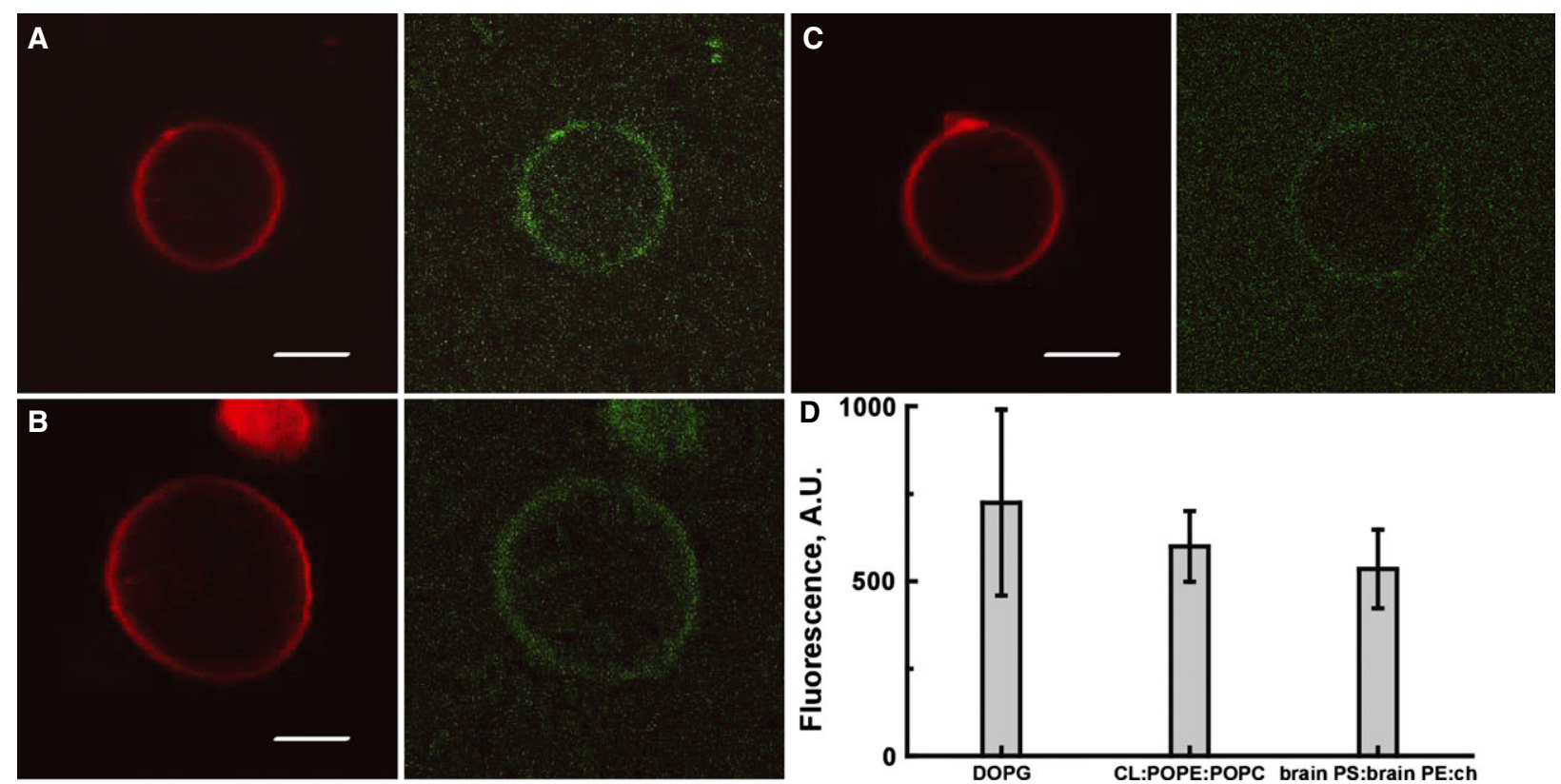

Fig. 3. Representative confocal microscopy images of DOPE-rhodamine labeled GUVs (red channel) and $\alpha \mathrm{S}$ wild-type-140C-A488 oligomers (green channel). Binding of $\alpha$ S oligomers to (A) DOPG, (B) CL : POPE : POPC and (C) brain PS : brain PE : cholesterol vesicles is depicted. (D) Semiquantitative analysis of fluorescently labeled oligomers bound to vesicles $(n=15)$. The error bars represent the SD. Scale bars $=5 \mu \mathrm{m}$. The same experimental settings (master gain, digital offset and laser power) were used for all images.

$[13,14,19,44-46]$. However, although most studies suggest that neutral membranes do not bind $\alpha \mathrm{S}[13,47]$, other studies claim differently [14,48]. The amount of $\alpha \mathrm{S}$ bound to lipid bilayers is proportional to the number of available binding sites and therefore to the

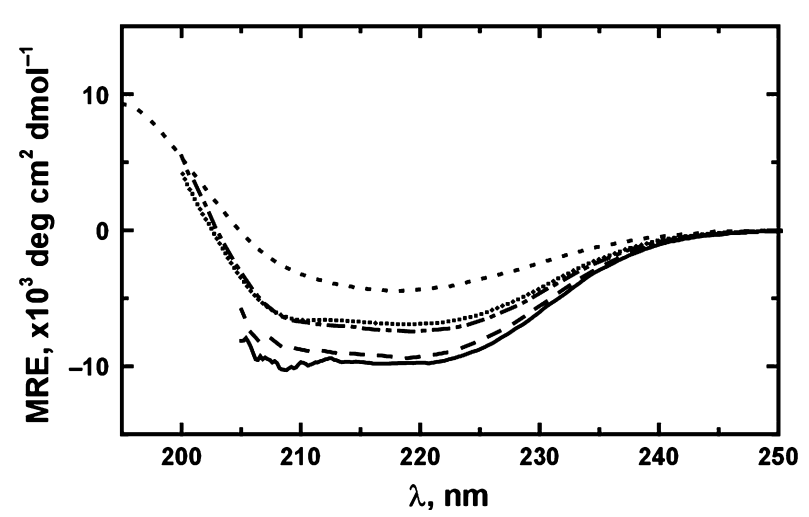

Fig. 4. $C D$ measurements of conformational changes of $\alpha S$ oligomers upon binding to membranes. Oligomers partially adopted $\alpha$-helical structure upon the addition of SUVs. No difference in changes of protein conformation was observed after $20 \mathrm{~h}$ for $\alpha \mathrm{S}$ oligomers binding to brain PS : brain PE : cholesterol vesicles. $\alpha S$ oligomers (...), DOPG (-. -), CL : POPE : POPC (...) and brain PS : brain PE : cholesterol after $10 \mathrm{~min}(--)$ and $20 \mathrm{~h}$ of incubation (-). fraction of negatively-charged lipids in the bilayer $[14,19]$. Earlier studies with brain-derived lipids showed no binding of $\alpha \mathrm{S}$ to lipid bilayers that contained only $10-15 \%$ of PS lipids, indicating that the $20 \%$ used in the present study is close to the minimal amount required for $\alpha \mathrm{S}$ binding [13]. Variations in composition of the brain PS : brain PE : cholesterol GUVs may explain why monomers did not bind to all brain PS : brain PE : cholesterol GUVs. This variation in composition is possibly caused by the presence of brain PS in the lipid mixture. It has been suggested that brain PS can interfere with the electroformation of GUVs resulting in variation in lipid composition of the resulting vesicles [49].

In the presence of excess vesicles of all lipid compositions (DOPG or natural lipids), $\alpha \mathrm{S}$ showed comparable results in $\mathrm{CD}$ binding studies. The maximal $\alpha$-helical content of the protein did not depend on the negatively-charged lipid species. Interactions of $\mathrm{CL}$ containing vesicles with $\alpha \mathrm{S}$ monomers have been characterized by ${ }^{19} \mathrm{~F}-\mathrm{NMR}$ [31]. It was proposed that the positively-charged $\mathrm{N}$-terminal region is involved in binding via an interaction between positivelycharged lysines and negatively-charged CL [31]. The protein binds to lipid surfaces through an amphipathic $\alpha$-helix adopted by 100 amino acid residues on the $\mathrm{N}$-terminal part of the protein, whereas the acidic 

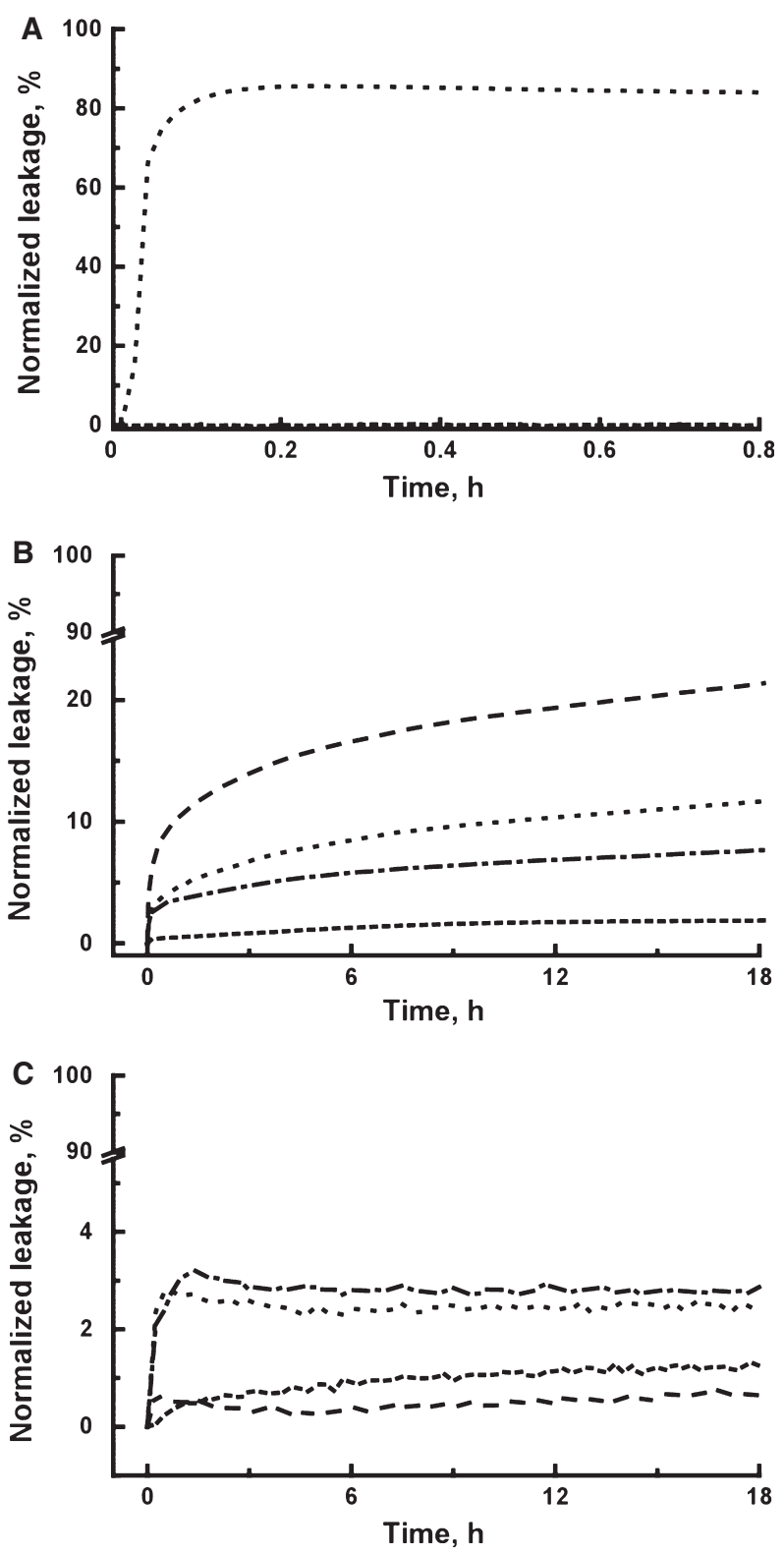

Fig. 5. Dye release kinetics from vesicles of different lipid composition after the addition of $\alpha \mathrm{S}$ oligomers. (A) with DOPG vesicles $1 \mu \mathrm{m}$ oligomers showed almost complete leakage and fast kinetics, which reached a plateau after $12 \mathrm{~min}$. (B) CL:POPE:POPC vesicles did not result in comparable leakage after $18 \mathrm{~h}$, even with $4 \mu \mathrm{M}$ oligomer concentration. (C) Oligomers did not induce appreciable leakage in brain PS: brain PE : cholesterol model membranes, even over a long time scale. $4 \mu \mathrm{M}$ oligomers $(---)$, $1 \mu \mathrm{m}$ oligomers (- - -), $0.5 \mu \mathrm{m}$ oligomers (- . -) and lipids alone (--).

C-terminal tail of the protein remains in solution [2]. According to the literature, $\alpha \mathrm{S}$ monomers adopt $41 \%$ $\alpha$-helix in the presence of $1 \mathrm{~mm}$ SDS [50], whereas, upon binding to DPPC : DPPG vesicles, $61 \%$ of the protein adopted the $\alpha$-helical conformation [51], which is comparable to the observations reported in the present study.

Similar to $\alpha \mathrm{S}$ monomers, oligomers were found to bind lipid bilayers of all compositions studied. However, compared to observations on $\alpha \mathrm{S}$ monomers, the helical content observed in the presence of oligomers was much lower [23]. It is possible that only the monomers in the oligomer facing the bilayer bind the membrane and adopt an $\alpha$-helical conformation. Alternatively, the interactions between $\alpha \mathrm{S}$ monomers in the oligomer may be too strong to permit further conformational changes upon lipid binding.

Oligomers interacting with simple negativelycharged lipid bilayers composed of, for example, DOPG or POPG immediately permeabilize these lipid membranes [25]. Fast content release was also observed for vesicles containing POPC : DOPA lipids $[23,43]$. The binding of oligomers to lipid bilayers has been reported to not always result in membrane permeabilization [23,25]. Even $30 \mathrm{~min}$ after the addition of the oligomers, POPG : POPC bilayers remained intact $[43,52]$. For the two-component lipid mixture CL : POPC (1:3), a lipid composition that comes close to that of mitochondrial membranes, no leakage was observed over short time scales [23]. In the present study, we show that the interaction of oligomers with bilayers does not necessarily result in a fast loss of membrane integrity. Membranes with a lipid composition [CL : POPE : POPC $(4: 3: 5)$ ] similar to that reported earlier [23] show some dye release over $18 \mathrm{~h}$ (Fig. 5B). However, even over these long time scales, oligomer binding did not result in permeabilization of the plasma membrane mimicking lipid bilayers (brain PS : brain PE : cholesterol) (Fig. 5C). To describe the oligomer-induced calcein release kinetics from CL : POPE : POPC vesicles, at least three exponentials, and therefore at least three rate constants, were necessary. The presence of more than one rate constant suggests that subpopulations of vesicles are differently affected by the addition of oligomers. The value of one of the rate constants $\left(k_{3}\right.$ in Table 1) describing the calcein flux as a function of oligomer concentration is comparable to that observed for lipid bilayers in the absence of oligomers $[53,54]$. This suggests that a fraction of the vesicles is not affected by the oligomers. Oligomers either did not bind or did not affect the calcein permeability of this fraction. As expected, the fraction of unaffected vesicles becomes smaller with an increasing oligomer concentration. The amplitude of the other two exponents increases with the oligomer concentration. The dye release kinetics described by these two exponents is therefore attributed to the presence of oligomers in 
Table 1. Fitting parameters for oligomer-induced calcein leakage from CL:POPE:POPC vesicles. Fits were determined using $y=A \times\left(1-\mathrm{e}^{-k_{1} \times \mathrm{t}}\right)+B \times\left(1-\mathrm{e}^{-\mathrm{k}_{2} \times \mathrm{t}}\right)+C \times\left(1-\mathrm{e}^{-\mathrm{k}_{3} \times \mathrm{t}}\right)+y_{0} . A$ and $B$, pre-exponential factors associated with oligomer-induced leakage; $k_{1}$ and $k_{2}$, rate constant of oligomer-induced leakage; $C$, pre-exponental factor of bare membrane leakage; $k_{3}$, rate constant bare vesicle leakage; $y_{0}$, the offset. We present calcein release kinetics measured for six different oligomer concentrations: $4,3,2,1,0.5$ and $0.25 \mu \mathrm{m}$. At least three exponentials were necessary for adequate fits of our data.

\begin{tabular}{lccllrr}
\hline Concentration $(\mu \mathrm{M})$ & \multicolumn{1}{c}{$A$} & \multicolumn{1}{c}{$B$} & $C$ & \multicolumn{1}{c}{$k_{1}\left(\mathrm{~h}^{-1}\right)$} & $k_{2}\left(\mathrm{~h}^{-1}\right)$ & $k_{3}\left(\mathrm{~h}^{-1}\right)$ \\
\hline 4 & $6.4 \pm 0.17$ & $7.8 \pm 0.13$ & 86 & $10.87 \pm 0.59$ & $0.47 \pm 0.02$ & 0.004 \\
3 & $6.33 \pm 0.14$ & $7.39 \pm 0.12$ & 86 & $10.15 \pm 0.47$ & $0.45 \pm 0.02$ & 0.004 \\
2 & $5.8 \pm 0.1$ & $7.5 \pm 0.08$ & 85.5 & $12.59 \pm 0.47$ & $0.47 \pm 0.012$ & 0.004 \\
1 & $2.87 \pm 0.06$ & $4.79 \pm 0.07$ & 92 & $10.58 \pm 0.48$ & $0.34 \pm 0.01$ & 0.002 \\
0.5 & $2.69 \pm 0.04$ & $2.69 \pm 0.07$ & 94.03 & $13.88 \pm 0.47$ & $0.31 \pm 0.01$ & 0.001 \\
0.25 & $2.52 \pm 0.04$ & $1.31 \pm 0.03$ & 96.4 & $13.28 \pm 0.4$ & $0.37 \pm 0.03$ & $<0.001$ \\
\hline
\end{tabular}

or on the lipid bilayer. Unexpectedly, both rate constants are observed to increase with increasing oligomer concentrations (Table 1). The oligomer-dependent exponents are expected to contain contributions of both the lipid bilayer and the oligomers. Because the leakage is much faster in the presence of oligomers, we assume that the contribution of oligomers is very large compared to the contribution of the bare bilayer. As indicated in the Results, the value of $k$ depends not only on the permeability coefficient $P$, but also on the volume of the vesicle and the area over which diffusion takes place. With increasing oligomer concentrations, the area covered by oligomers is expected to become larger. In contrast to

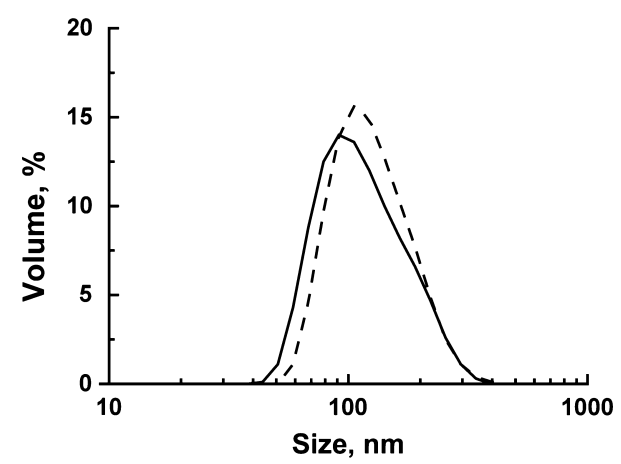

Fig. 6. Typical dynamic light scattering graph for CL : POPE : POPC LUVs before and after the addition of oligomers. Parameters from dynamic light scattering measurements are described as the volume of particles (\%) as a function of size $(\mathrm{nm})$ [F(size, $\mathrm{nm})=$ volume of vessicles, \%] for vesicles alone [CL : POPE : POPC (-)] and vesicles + oligomers $(--)$. Changes in diameter between membranes $(144.1 \pm 6.0)$ and membranes after the addition of oligomers $(177.8 \pm 15.1)$ are approximately $30 \mathrm{~nm}$ for $\mathrm{CL}$ : POPE : POPC membranes. Peak width changes were approximately $12 \mathrm{~nm}$; peak width of vesicles alone is $50.2 \pm 6.5 \mathrm{~nm}$ and peak width after $18 \mathrm{~h}$ of incubation with oligomers is $62.6 \pm 4.5 \mathrm{~nm}$. our observation, this increase in oligomer area should give rise to an increase in the rate constants contributed to oligomers. The observed decrease in the rate constants may result from an increase in vesicle volume (i.e. by incorporation of oligomers in the lipid bilayer). Dynamic light scattering experiments indicate that CL: POPE : POPC LUVs indeed become larger upon incubation with oligomers, supporting the hypothesis that the decrease in rate constants corresponding to oligomer-induced leakage results from a volume increase. The observed change in the CL: POPE : POPC vesicle diameter of approximately $30 \mathrm{~nm}$ corresponds to the expected volume change resulting from oligomer incorporation (Fig. 6). The decrease in leakage observed for brain PS : brain PE : cholesterol bilayers in the presence of oligomers possibly results from the binding of oligomers and blocking of the membrane surface. The permeability of the oligomer-covered surface is low compared to the permeability of the bare membrane surface.

Recent data on more physiologically relevant brain total lipid extract membranes (BTLEMs) have shown that $\alpha \mathrm{S}$ causes bilayer defects [55]. We speculate that the brain PS : brain PE : cholesterol model system that we use may show a lipid rearrangement similar to that proposed for BTLEMs. Cholesterol in complex model brain PS : brain PE : cholesterol membranes can have an additional stabilizing effect on these membranes $[23,43,48,56]$, making them less vulnerable to oligomer-induced leakage. In our experimental system, the inclusion of cholesterol at a constant charge density does not appear to have any appreciable effect on the extent of permeabilization (Fig. 7). However, it has been suggested that the amount of cholesterol in the plasma membrane is important for the formation of amyloid channels $[57,58]$. 


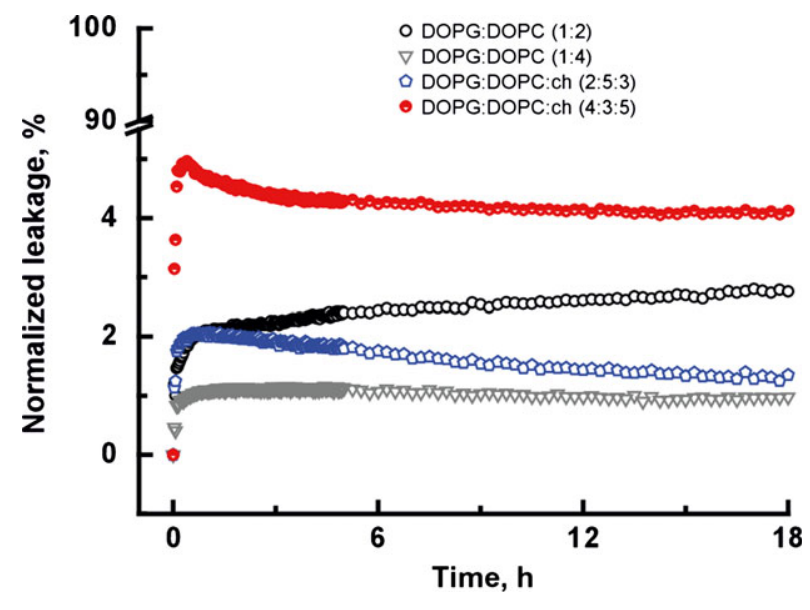

Fig. 7. Oligomer-induced leakage kinetics from vesicles of different lipid composition: DOPG : DOPC (1:2, black circles), DOPG : DOPC (1:4, grey triangles), DOPG : DOPC:cholesterol (2:5:3, blue pentagons) and DOPG : DOPC : cholesterol $(4: 3: 5$, red circles). Oligomers at $1 \mu \mathrm{M}$ (equivalent monomer) concentration were incubated with the lipid membranes for $18 \mathrm{~h}$. Oligomer-induced leakage was $<5 \%$, which is comparable to the data observed with our plasma inner leaflet membrane mimics.

For the amyloid-forming protein islet amyloid polypeptide, slow dye release from POPG : POPC vesicles was concluded to be caused by the formation of fibrils [59]. The experiments reported in the present study were performed with stable oligomers [60]. Thus, we do not expect that conformational changes and further aggregation play a role in long-term leakage kinetics. Alternatively, any conformational changes in the oligomer may be beyond our detection limit because they involve only a few proteins.

The reported membrane leakage data suggest that complex lipid membranes are less prone to oligomerinduced damage. The complexity of model plasma membranes lies in the composition of the membranes itself, which consist largely of combinations of long and polyunsaturated lipids in the brain lipid extracts used. The results of the present study show that mitochondrial model membranes are more prone to oligomer-induced damage over longer time scales, whereas the more complex plasma membrane model systems do not show a concentration-dependent permeabilization over the same time scale. Other in vitro studies have suggested that the mitochondria specific lipid CL is essential for $\alpha \mathrm{S}$ mitochondrial membrane interactions [46]. A recent study [61] confirmed a common mechanism of damage for mitochondrial membranes by amyloid-induced species via direct interactions of these species with membrane phospholipids. Their proposed mechanism agrees very well with our data on mitochondrial model membranes, which is mediated by the increased affinity of these species for CL.

\section{Materials and methods}

\section{Expression and purification of $\alpha \mathrm{S}$}

Expression and purification of human wild-type $\alpha \mathrm{S}$ and the cysteine (Cys) mutant $\alpha \mathrm{S}-\mathrm{A} 140 \mathrm{C}$, where alanine at position 140 was replaced with a cysteine, was carried out as described previously [62]. The protein concentration was determined by measuring the absorbance on a spectrophotometer at $276 \mathrm{~nm}$, using molar extinction coefficients of $5600 \mathrm{M}^{-1} \cdot \mathrm{cm}^{-1}$ for wild-type and $5745 \mathrm{M}^{-1} \cdot \mathrm{cm}^{-1}$ for A140C $[60,63]$. The protein was stored at $-80{ }^{\circ} \mathrm{C}$ until further use.

\section{Labeling of $\alpha \mathbf{S}$}

The Cys mutant $\alpha \mathrm{S}-\mathrm{A} 140 \mathrm{C}$ was used for labeling the protein with an Alexa Fluor 488 C5 maleimide dye (A488) (Invitrogen, Carlsbad, CA, USA). Prior to labeling, a six-fold molar excess of dithiothreitol was added to $\alpha \mathrm{S}-\mathrm{A} 140 \mathrm{C}$ to reduce disulfide bonds. After $30 \mathrm{~min}$ of incubation, dithiothreitol was removed using Zeba Spin desalting columns (Pierce, Rockford, IL, USA) and a two-fold excess of A488 was added. After $1 \mathrm{~h}$ of incubation, excess of free dye was removed using two desalting steps. The labeling efficiency was estimated to be in the range $90-100 \%$ from absorption spectra. To determine the protein and A488 concentration the absorbance at $276 \mathrm{~nm}$ was measured using a molar extinction coefficient of $5745 \mathrm{M}^{-1} \cdot \mathrm{cm}^{-1}$ for the protein and at $495 \mathrm{~nm}$ using a molar extinction coefficient of $72000 \mathrm{~m}^{-1} \cdot \mathrm{cm}^{-1}$ for the dye.

\section{Preparation of unlabeled and labeled $\alpha S$ oligomers}

Briefly, oligomers were obtained by incubating $\alpha \mathrm{S}$ at high concentrations in the absence of additional factors [23]. Alexa 488 labeled oligomers with $7.5 \%$ labeling density, achieved by mixing appropriate quantities of labeled protein $(\alpha \mathrm{S}-\mathrm{A} 140 \mathrm{C})$ with unlabeled protein (wild-type), were prepared for membrane binding studies by confocal microscopy. Oligomers were purified and separated from monomers using size-exclusion chromatography on a Superdex ${ }^{\mathrm{TM}} 200$ 10/300 GL column (GE Healthcare Bio-Sciences AB, Uppsala, Sweden). Separation of oligomers from monomers is based on size, where larger particles (oligomers) elute first. To confirm the presence of oligomers, a native PAGE gradient gel was used [23] with a polyacrylamide gradient of between $3 \%$ and $16 \%$. We have previously demonstrated that oligomers prepared in this manner are composed of approximately 30 monomers, and are stable [60]. Monomers could not be detected in the oligomer preparation (data not shown). 


\section{LUV preparation and calcein release assay}

All lipids were purchased from Avanti Polar Lipids (Alabaster, AL, USA). In the experiments the lipids used were: POPC, DOPG, POPE, 1',3'-bis[1,2-dioleoyl-sn-glycero-3phospho]-sn-glycerol (18: $1 \mathrm{CL}$ ), porcine brain L- $\alpha$-phosphatidylserine (brain PS), porcine brain L- $\alpha$-phosphatidylethanolamine (brain PE) and cholesterol.

To mimic the lipid composition of neuronal plasma membranes, we used mixtures of brain PS : brain PE : cholesterol in a molar ratio of $2: 5: 3$, which corresponds to $20 \%$ of negatively-charged lipids. However, no elaborate data of brain lipid compositions are available. The neuronal membrane has approximately $10 \%$ negatively-charged lipids, mainly PS, whereas the main components of these membranes are neutral PC and PE phospholipids [64]. However, this estimation of the lipid composition does not take into account asymmetry between the inner and outer leaflets of the membrane [13]. Because $\alpha \mathrm{S}$ is an intracellular protein, we chose to the mimic the inner leaflet of the plasma membrane, which is enriched in PS. $\alpha \mathrm{S}$ has also been implicated in interactions with mitochondrial membranes. Data from the literature suggest that $\alpha \mathrm{S}$ preferentially binds to the mitochondrial inner membrane [29]. To mimic the mitochondrial inner membrane, we used a lipid composition that contains CL : POPE : POPC in a molar ratio $4: 3: 5$ [29].

Specific lipid compositions were prepared by mixing $650 \mu \mathrm{M}$ of lipids in chloroform. The solvent was removed by drying under nitrogen flow. The resulting lipid films were then hydrated for $1 \mathrm{~h}$ using $50 \mathrm{~mm}$ calcein, $10 \mathrm{~mm}$ Hepes and $60 \mathrm{~mm} \mathrm{NaCl}$ to obtain an osmolality (Cryoscopic osmometer; Gonotec, Berlin, Germany) of $320 \mathrm{mOsm} \cdot \mathrm{kg}^{-1}$. The sample was then subjected to five freeze-thaw cycles using liquid nitrogen and a water bath. The temperature of the water bath was kept above the transition temperature of the lipid mixture. The solution was subsequently extruded 11 times through 100 -nm pore size filters (Whatman, Maidstone, UK) and finally unencapsulated calcein was removed using PD-10 columns filled with Sephadex G-100 (GE Healthcare Bio-Sciences AB).

Long-term calcein release kinetics of the model membranes was followed on a Varian Cary Eclipse fluorometer (Varian Inc., Palo Alto, CA, USA). We used lipid concentrations of $40 \mu \mathrm{M}$. The emission intensity was recorded at $515 \mathrm{~nm}$ for excitation at $495 \mathrm{~nm}$. Triton X (0.5\%) was added to completely lyse the vesicles. All the data points were normalized using the intensity after Triton $\mathrm{X}$ treatment as $100 \%$ leakage.

\section{Semi-quantitative $\alpha$ S monomer and oligomer binding assay}

GUVs were prepared in sucrose solution as described previously by Angelova and Dimitrov [65]. The sucrose concen- tration was equiosmolar to the $10 \mathrm{~mm}$ Hepes, $150 \mathrm{~mm}$ $\mathrm{NaCl}$ solution in which the proteins were dissolved. $1 \%$ DOPE-rhodamine was included in the lipid mixtures to facilitate visualization of the lipid membrane.

GUVs mimicking natural lipid compositions were equilibrated with fluorescently labeled oligomers for $30 \mathrm{~min}$ before imaging. DOPG vesicles were used as a positive control for binding of monomers and oligomers to membranes.

The experiments were performed using the same experimental settings (master gain, digital offset and laser power) for imaging the binding of both monomers (Fig. 1) and oligomers (Fig. 3). DOPE-rhodamine was excited with a $\mathrm{He} / \mathrm{Ne}$ laser $(543 \mathrm{~nm})$ and Alexa 488 was excited with an Argon laser $(488 \mathrm{~nm})$ on a CLSM 510 confocal microscope (Carl Zeiss, Oberkochen, Germany). We used the script plot profile in IMAGEJ (NIH, Bethesda, MD, USA) to extract semi-quantitative fluorescence values reporting on the binding of monomers and oligomers to membranes. The amount of $\alpha \mathrm{S}$ bound to GUV membranes was estimated from the peak values of the Alexa 488 intensity profiles and averaged for at least 15 vesicles on five random cross-sections using the same imaging settings (master gain, digital offset and laser power). Figures $1 \mathrm{~A}-\mathrm{C}$ and $3 \mathrm{~A}-\mathrm{C}$ show representative confocal images of the binding of fluorescently labeled $\alpha \mathrm{S}$ monomers (Fig. 1) and oligomers (Fig. 3).

\section{SUVs preparation and binding of $\alpha \mathrm{S}$ oligomers to SUVs}

Lipid mixtures that contain $3.8 \mathrm{~mm}$ of lipids in chloroform were prepared. After removing traces of chloroform, a thin lipid film was dissolved in $10 \mathrm{~mm}$ K-phosphate buffer. SUVs were prepared from this solution by sonicating for $30 \mathrm{~min}$ using a tip sonicator (Labsonic, Branson Ultrasonics, Danbury, CT, USA) at maximum amplitude on ice. Binding-induced conformational changes of oligomeric and monomeric $\alpha \mathrm{S}$ were investigated using $\mathrm{CD}$ spectroscopy [66]. Both protein and lipids samples were prepared in $10 \mathrm{~mm}$ K-phosphate buffer. Spectra were recorded using a Jasco 715 spectropolarimeter (Jasco Inc., Easton, MD, USA) in the range $190-260 \mathrm{~nm}$ with a step size of $0.2 \mathrm{~nm}$, band width $2 \mathrm{~nm}$ and scanning speed $50 \mathrm{~nm} \cdot \mathrm{min}^{-1}$ in 1$\mathrm{mm}$ quartz cuvettes. The spectra represent the mean of three scans and were corrected for the background from $\mathrm{K}$-phosphate buffer and $\alpha \mathrm{S}$-free vesicles. Monomer and oligomer concentrations were 16 and $10 \mu \mathrm{M}$ of equivalent monomer concentration, respectively, keeping the lipid to protein ratio $260: 1$ for oligomers binding to SUVs and $170: 1$ for monomers binding to SUVs. A lipid concentration of $2.72 \mathrm{~mm}$ resulted in the above-mentioned lipid to protein ratio. These ratios were chosen to obtain complete binding of the protein $[13,42]$. MREs $\left(\mathrm{deg} \cdot \mathrm{dmol}^{-1} \cdot \mathrm{cm}^{-2}\right)$ were calculated using Eqn (1), where $c$ is the protein concentration, $l$ is the pathlength cuvette and $n_{\text {residues }}$ is the number of residues (amino acids): 


$$
M R E=\frac{\text { recorded value }- \text { buffer value }}{l(\mathrm{~cm}) \times n_{\text {residues }} \times 10 \times c(M)}
$$

In principle, the MRE at any wavelength is a combination of $\alpha$-helical structure, $\beta$-sheet and random coil content of the sample [67]. The $\alpha$-helical content was estimated by measuring the $C D$ signal at $222 \mathrm{~nm}$; at this wavelength, the contribution of random coil structure is relatively small [68].

At $222 \mathrm{~nm}$, the $\alpha$-helical content was obtained using Eqns $(2,3,4)$ :

$$
\% \text { helicity }=\frac{\theta_{222}-\theta_{\text {coil }}}{\theta_{\text {helix }}-\theta_{\text {coil }}} \times 100
$$

where $\theta_{\text {helix }}$ and $\theta_{\text {coil }}$ could be calculated:

$$
\begin{gathered}
\theta_{\text {coil }}=640-45 \times \vartheta \\
\theta_{\text {helix }}=-4000 \times\left(1-\frac{2.5}{n}\right)+100 \times \vartheta
\end{gathered}
$$

where $\Theta_{222}$ is the measured mean residue ellipticity at $222 \mathrm{~nm}, \theta_{\text {helix }}$ and $\theta_{\text {coil }}$ are mean residue ellipticities at $222 \mathrm{~nm}$ of idealized $\alpha$-helix and random coil proteins, $n=140$ amino acids and $\vartheta$ is the temperature $\left({ }^{\circ} \mathrm{C}\right)$.

\section{Acknowledgements}

We thank Kirsten van Leijenhorst-Groener and Nathalie Schilderink for assistance with the expression and purification of $\alpha$-synuclein. This work was financially supported by the Nederlandse Organisatie voor Wetenschappelijk Onderzoek (NWO) via NWO-CW TOP grant number 700.58.302. M. T. S. and V. S. acknowledge support by NanoNextNL, a micro and nanotechnology consortium of the Government of the Netherlands and 130 partners.

\section{Author contributions}

A.N.D.S. planned and performed the experiments, and also analyzed the data. All authors contributed to interpreting the data and writing the manuscript.

\section{References}

1 Cookson MR (2009) alpha-Synuclein and neuronal cell death. Mol Neurodegener 4, 9.

2 Eliezer D, Kutluay E, Bussell R Jr \& Browne G (2001) Conformational properties of alpha-synuclein in its free and lipid-associated states. J Mol Biol 307, 1061-1073.
3 Uversky VN, Li J \& Fink AL (2001) Evidence for a partially folded intermediate in alpha-synuclein fibril formation. J Biol Chem 276, 10737-10744.

4 Lorenzen N, Nielsen SB, Buell AK, Kaspersen JD, Arosio P, Vad BS, Paslawski W, Christiansen G, Valnickova-Hansen Z, Andreasen M et al. (2014) The role of stable $\alpha$-synuclein oligomers in the molecular events underlying amyloid formation. J Am Chem Soc 136, 3859-3868.

5 Roche J, Ying JF, Maltsev AS \& Bax A (2013) Impact of hydrostatic pressure on an intrinsically disordered protein: a high-pressure NMR study of alpha-synuclein. ChemBioChem 14, 1754-1761.

6 Zanzoni A, Marchese D, Agostini F, Bolognesi B, Cirillo D, Botta-Orfila M, Livi CM, Rodriguez-Mulero S \& Tartaglia GG (2013) Principles of self-organization in biological pathways: a hypothesis on the autogenous association of alpha-synuclein. Nucleic Acids Res 41, 9987-9998.

7 Breydo L, Wu JW \& Uversky VN (2012) Alphasynuclein misfolding and Parkinson's disease. Biochim Biophys Acta 1822, 261-285.

8 Uversky VN (2007) Neuropathology, biochemistry, and biophysics of alpha-synuclein aggregation. $J$ Neurochem 103, 17-37.

9 Auluck PK, Caraveo G \& Lindquist S (2010) alphaSynuclein: membrane interactions and toxicity in Parkinson's disease. Annu Rev Cell Dev Biol 26, 211233.

10 Kayed R, Head E, Thompson JL, McIntire TM, Milton SC, Cotman CW \& Glabe CG (2003) Common structure of soluble amyloid oligomers implies common mechanism of pathogenesis. Science 300, 486-489.

11 Kayed R, Sokolov Y, Edmonds B, McIntire TM, Milton SC, Hall JE \& Glabe CG (2004)

Permeabilization of lipid bilayers is a common conformation-dependent activity of soluble amyloid oligomers in protein misfolding diseases. $\mathrm{J}$ Biol Chem 279, 46363-46366.

12 Jao CC, Hegde BG, Chen J, Haworth IS \& Langen R (2008) Structure of membrane-bound alpha-synuclein from site-directed spin labeling and computational refinement. Proc Natl Acad Sci USA 105, 19666-19671.

13 Davidson WS, Jonas A, Clayton DF \& George JM (1998) Stabilization of alpha-synuclein secondary structure upon binding to synthetic membranes. $J$ Biol Chem 273, 9443-9449.

14 Rhoades E, Ramlall TF, Webb WW \& Eliezer D (2006) Quantification of alpha-synuclein binding to lipid vesicles using fluorescence correlation spectroscopy. Biophys J 90, 4692-4700.

15 Middleton ER \& Rhoades E (2010) Effects of curvature and composition on alpha-synuclein binding to lipid vesicles. Biophys J 99, 2279-2288. 
16 Shvadchak VV, Yushchenko DA, Pievo R \& Jovin TM (2011) The mode of alpha-synuclein binding to membranes depends on lipid composition and lipid to protein ratio. FEBS Lett 585, 3513-3519.

17 Kjaer L, Giehm L, Heimburg T \& Otzen D (2009) The influence of vesicle size and composition on alphasynuclein structure and stability. Biophys $J$ 96, 28572870.

18 Pandey AP, Haque F, Rochet JC \& Hovis JS (2009) Clustering of alpha-synuclein on supported lipid bilayers: role of anionic lipid, protein, and divalent ion concentration. Biophys $J$ 96, 540-551.

19 Stockl M, Fischer P, Wanker E \& Herrmann A (2008) Alpha-synuclein selectively binds to anionic phospholipids embedded in liquid-disordered domains. J Mol Biol 375, 1394-1404.

20 Danzer KM, Haasen D, Karow AR, Moussaud S, Habeck M, Giese A, Kretzschmar H, Hengerer B \& Kostka M (2007) Different species of alpha-synuclein oligomers induce calcium influx and seeding. J Neurosci 27, 9220-9232.

21 Cappai R, Leck SL, Tew DJ, Williamson NA, Smith DP, Galatis D, Sharples RA, Curtain CC, Ali FE, Cherny RA et al. (2005) Dopamine promotes alphasynuclein aggregation into SDS-resistant soluble oligomers via a distinct folding pathway. FASEB J 19, 1377-1379.

22 Lashuel HA, Petre BM, Wall J, Simon M, Nowak RJ, Walz T \& Lansbury PT Jr (2002) Alpha-synuclein, especially the Parkinson's disease-associated mutants, forms pore-like annular and tubular protofibrils. $J \mathrm{Mol}$ Biol 322, 1089-1102.

23 van Rooijen BD, Claessens MM \& Subramaniam V (2009) Lipid bilayer disruption by oligomeric alphasynuclein depends on bilayer charge and accessibility of the hydrophobic core. Biochim Biophys Acta 1788, 1271-1278.

24 van Rooijen BD, Claessens MM \& Subramaniam V (2010) Membrane permeabilization by oligomeric asynuclein: in search of the mechanism. PLoS One 13, e14292.

25 Volles MJ, Lee SJ, Rochet JC, Shtilerman MD, Ding TT, Kessler JC \& Lansbury PT Jr (2001) Vesicle permeabilization by protofibrillar alpha-synuclein: implications for the pathogenesis and treatment of Parkinson's disease. Biochemistry 40, 7812-7819.

26 Meratan AA, Ghasemi A \& Nemat-Gorgani M (2011) Membrane integrity and amyloid cytotoxicity: a model study involving mitochondria and lysozyme fibrillation products. $J$ Mol Biol 409, 826-838.

27 Nakamura K, Nemani VM, Azarbal F, Skibinski G, Levy JM, Egami K, Munishkina L, Zhang J, Gardner B, Wakabayashi J et al. (2011) Direct membrane association drives mitochondrial fission by the
Parkinson disease-associated protein alpha-synuclein. $J$ Biol Chem 286, 20710-20726.

28 Yong-Kee CJ, Sidorova E, Hanif A, Perera G \& Nash JE (2012) Mitochondrial dysfunction precedes other sub-cellular abnormalities in an in vitro model linked with cell death in Parkinson's disease. Neurotox Res 21, 185-194.

29 Ardail D, Privat JP, Egret-Charlier M, Levrat C, Lerme F \& Louisot P (1990) Mitochondrial contact sites. Lipid composition and dynamics. J Biol Chem 265, 18797-18802.

30 Ramakrishnan M, Jensen PH \& Marsh D (2003) Alpha-synuclein association with phosphatidylglycerol probed by lipid spin labels. Biochemistry 42, 1291912926.

31 Zigoneanu IG, Yang YJ, Krois AS, Haque E \& Pielak GJ (2012) Interaction of alpha-synuclein with vesicles that mimic mitochondrial membranes. Biochim Biophys Acta 1818, 512-519.

32 Liu G, Zhang C, Yin J, Li X, Cheng F, Li Y, Yang H, Ueda K, Chan P \& Yu S (2009) alpha-Synuclein is differentially expressed in mitochondria from different rat brain regions and dose-dependently down-regulates complex I activity. Neurosci Lett 454, 187-192.

33 Li WW, Yang R, Guo JC, Ren HM, Zha XL, Cheng JS \& Cai DF (2007) Localization of a-synuclein to mitochondria within midbrain of mice. NeuroReport 18, 1543-1546.

34 Parihar MS, Parihar A, Fujita M, Hashimoto M \& Ghafourifar P (2008) Mitochondrial association of alpha-synuclein causes oxidative stress. Cell Mol Life Sci 65, 1272-1284.

35 Devi L, Raghavendran V, Prabhu BM, Avadhani NG \& Anandatheerthavarada HK (2008) Mitochondrial import and accumulation of alpha-synuclein impair complex I in human dopaminergic neuronal cultures and Parkinson disease brain. J Biol Chem 283, 90899100.

36 Devi L \& Anandatheerthavarada HK (2010) Mitochondrial trafficking of APP and alpha synuclein: relevance to mitochondrial dysfunction in Alzheimer's and Parkinson's diseases. Biochim Biophys Acta 1802, 11-19.

37 Guardia-Laguarta C, Area-Gomez E, Rub C, Liu Y, Magrane J, Becker D, Voos W, Schon EA \& Przedborski S (2014) alpha-Synuclein is localized to mitochondria-associated ER membranes. J Neurosci 34, 249-259.

38 Fauvet B, Fares MB, Samuel F, Dikiy I, Tandon A, Eliezer D \& Lashuel HA (2012) Characterization of semisynthetic and naturally Nalpha-acetylated alphasynuclein in vitro and in intact cells: implications for aggregation and cellular properties of alpha-synuclein. $J$ Biol Chem 287, 28243-28262. 
39 Gitler AD, Bevis BJ, Shorter J, Strathearn KE, Hamamichi S, Su LJ, Caldwell KA, Caldwell GA, Rochet JC, McCaffery JM et al. (2008) The Parkinson's disease protein alpha-synuclein disrupts cellular Rab homeostasis. Proc Natl Acad Sci USA 105, 145-150.

40 Fiske M, White M, Valtierra S, Herrera S, Solvang K, Konnikova A \& Debburman S (2011) Familial Parkinson's disease mutant E46K alpha-synuclein localizes to membranous structures, forms aggregates, and induces toxicity in yeast models. ISRN Neurol 2011, 521847.

41 Pacheco C, Aguayo LG \& Opazo C (2012) An extracellular mechanism that can explain the neurotoxic effects of alpha-synuclein aggregates in the brain. Front Physiol 3, 297.

42 Jo E, McLaurin J, Yip CM, St George-Hyslop P \& Fraser PE (2000) alpha-Synuclein membrane interactions and lipid specificity. J Biol Chem 275, 34328-34334.

43 van Rooijen BD, Claessens MM \& Subramaniam V (2008) Membrane binding of oligomeric alpha-synuclein depends on bilayer charge and packing. FEBS Lett $\mathbf{5 8 2}$, 3788-3792.

44 Zhu M, Li J \& Fink AL (2003) The association of alpha-synuclein with membranes affects bilayer structure, stability, and fibril formation. J Biol Chem 278, 40186-40197.

45 Wang GF, Li C \& Pielak GJ (2010) 19F NMR studies of alpha-synuclein-membrane interactions. Protein Sci 19, 1686-1691.

46 Zigoneanu IG, Yang YJ, Krois AS, Haque E \& Pielak GJ (2012) Interaction of alpha-synuclein with vesicles that mimic mitochondrial membranes. Biochim Biophys Acta 1818, 512-519.

47 Pfefferkorn CM \& Lee JC (2010) Tryptophan probes at the alpha-synuclein and membrane interface. $J$ Phys Chem B 114, 4615-4622.

48 Shvadchak VV, Falomir-Lockhart LJ, Yushchenko DA \& Jovin TM (2011) Specificity and kinetics of alphasynuclein binding to model membranes determined with fluorescent excited state intramolecular proton transfer (ESIPT) probe. J Biol Chem 286, 13023-13032.

49 Schwiering M \& Hellmann N (2012) Validation of liposomal lipid composition by thin-layer chromatography. J Liposome Res 22, 279-284.

50 Chandra S, Chen X, Rizo J, Jahn R \& Sudhof TC (2003) A broken alpha -helix in folded alpha -Synuclein. J Biol Chem 278, 15313-15318.

51 Bartels T, Ahlstrom LS, Leftin A, Kamp F, Haass C, Brown MF \& Beyer K (2010) The N-terminus of the intrinsically disordered protein alpha-synuclein triggers membrane binding and helix folding. Biophys $J \mathbf{9 9}$, 2116-2124.
52 Volles M \& Lansbury P (2002) Vesicle permeabilization by protofibrillar alpha-synuclein is sensitive to Parkinson's disease-linked mutations and occurs by a pore-like mechanism. Biochemistry 41, 4595-4602.

53 Maherani B, Arab-Tehrany E, Kheirolomoom A, Geny D \& Linder M (2013) Calcein release behavior from liposomal bilayer; influence of physicochemical/ mechanical/structural properties of lipids. Biochimie $\mathbf{9 5}$, 2018-2033.

54 Shimanouchi T, Ishii H, Yoshimoto N, Umakoshi H \& Kuboi R (2009) Calcein permeation across phosphatidylcholine bilayer membrane: effects of membrane fluidity, liposome size, and immobilization. Colloids Surf B Biointerfaces 73, 156-160.

55 Ouberai MM, Wang J, Swann MJ, Galvagnion C, Guilliams T, Dobson CM \& Welland ME (2013) alphaSynuclein senses lipid packing defects and induces lateral expansion of lipids leading to membrane remodeling. J Biol Chem 288, 20883-20895.

56 Leftin A, Job C, Beyer K \& Brown MF (2013) Solidstate $13 \mathrm{C}$ NMR reveals annealing of raft-like membranes containing cholesterol by the intrinsically disordered protein $\alpha$-synuclein. J Mol Biol 425, 2973 2987.

57 Fantini J \& Yahi N (2013) The driving force of alphasynuclein insertion and amyloid channel formation in the plasma membrane of neural cells: key role of ganglioside- and cholesterol-binding domains. Adv Exp Med Biol 991, 15-26.

58 Fantini J, Carlus D \& Yahi N (2011) The fusogenic tilted peptide (67-78) of alpha-synuclein is a cholesterol binding domain. Biochim Biophys Acta 1808, 23432351.

59 Brender JR, Lee EL, Hartman K, Wong PT, Ramamoorthy A, Steel DG \& Gafni A (2011) Biphasic effects of insulin on islet amyloid polypeptide membrane disruption. Biophys J 100, 685-692.

60 Zijlstra N, Blum C, Segers-Nolten IM, Claessens MM \& Subramaniam V (2012) Molecular composition of sub-stoichiometrically labeled alpha-synuclein oligomers determined by single-molecule photobleaching. Angew Chem Int Ed Engl 51, 8821-8824.

61 Camilleri A, Zarb C, Caruana M, Ostermeier U, Ghio S, Hogen T, Schmidt F, Giese A \& Vassallo N (2013) Mitochondrial membrane permeabilisation by amyloid aggregates and protection by polyphenols. Biochim Biophys Acta 1828, 2532-2543.

62 van Raaij ME, Segers-Nolten IMJ \& Subramaniam V (2006) Quantitative morphological analysis reveals ultrastructural diversity of amyloid fibrils from $\alpha$ synuclein mutants. Biophys $J$ 91, L96-L98.

63 Pace CN, Vajdos F, Fee L, Grimsley G \& Gray T (1995) How to measure and predict the molar absorption coefficient of a protein. Protein Sci 4, 24112423 . 
64 van Meer G, Voelker DR \& Feigenson GW (2008) Membrane lipids: where they are and how they behave. Nat Rev Mol Cell Biol 9, 112-124.

65 Angelova MI \& Dimitrov DS (1986) Liposome electroformation. Faraday Disc Chem Soc 81, 303-311.

66 Barrow CJ, Yasuda A, Kenny PT \& Zagorski MG (1992) Solution conformations and aggregational properties of synthetic amyloid beta-peptides of Alzheimer's disease. J Mol Biol 225, 1075-1093.
67 Chen YH, Yang JT \& Chau KH (1974) Determination of the helix and beta form of proteins in aqueous solution by circular dichroism. Biochemistry 13, 33503359.

68 Scholtz JM, Qian H, York EJ, Stewart JM \& Baldwin RL (1991) Parameters of helix-coil transition theory for alanine-based peptides of varying chain lengths in water. Biopolymers 31, 1463-1470. 